\title{
ORIGINAL RESEARCH \\ Tract-Based Spatial Statistics of Diffusion Tensor Imaging in Adults with Dyslexia
}

T. Richards

J. Stevenson

J. Crouch

L.C. Johnson

K. Maravilla

P. Stock

R. Abbott

V. Berninger
BACKGROUND AND PURPOSE: Diffusion tensor imaging is a tool that can be used to study white matter microstructure in dyslexia. We tested the hypothesis that dyslexics have a white matter structural change (as measured by directional diffusion of water, which can be affected by disruption in white matter tracts) between brain regions that previous functional connectivity studies showed were associated with phonologic processing.

MATERIALS AND METHODS: Diffusion tensor imaging (DTI) scans were acquired from 7 healthy adult normal readers and from 14 adults with dyslexia on a 1.5T scanner. Voxelwise statistical analysis of the fractional anisotropy data were carried out by using Tract-Based Spatial Statistics to compare dyslexic subjects versus control subjects in white matter tracts.

RESULTS: Significant group difference map clusters (comparing adults with and without dyslexia) occurred in specific bilateral white matter tracts within the frontal lobe, temporal lobe, occipital lobe, and parietal lobe.

CONCLUSION: The DTI fractional anisotropy results in the bilateral white matter showing higher fractional anisotropy in adult control subjects compared with adults with dyslexia (relating to white matter fiber tract integrity) are consistent with our previous functional connectivity results from seed points in the bilateral inferior frontal gyrus.
D iffusion tensor imaging (DTI) is a technique that can be used to study white matter structures (anisotropy) in the human brain. ${ }^{1}$ Le Bihan et $\mathrm{al}^{2}$ have written a review about the applications of DTI to study abnormalities in a variety of diseases including stroke, multiple sclerosis, dyslexia, and schizophrenia. DTI has recently been used to study language-related neural pathways in normal and impaired readers. ${ }^{1-7}$ Klingberg et $\mathrm{al}^{5}$ reported that white matter diffusion anisotropy in the temporoparietal region of the left hemisphere was significantly correlated with reading scores within the reading-impaired group and within the control group in adults. Niogi and McCandliss ${ }^{7}$ used DTI to investigate the association between variation in white matter microstructure and individual differences in reading skill within children. Their results replicate previous findings of strong positive correlations between fractional anisotropy (FA) values in left centrum semiovale, left superior corona radiate, and bilateral anterior corona radiate and standardized reading scores of typically developing children. ${ }^{7}$ Deutsch et $\mathrm{al}^{3}$ investigated the white matter structure in the left temporoparietal region in children with a wide range of reading performance levels by using DTI. They report that the white matter structure measured using FA and coherence index correlated significantly with behavioral measurements of reading, spelling, and rapid naming performance. ${ }^{3}$

Molko et $\mathrm{al}^{6}$ used DTI to study a patient with alexia in his

Received November 20, 2007; accepted after revision December 20.

From the Department of Radiology (T.R., J.S., K.M.), Psychosocial and Community Health, School of Nursing (L.C.J.), and Educational Psychology (P.S., R.A., V.B.), University of Washington, Seattle, Wash; School of Medicine (J.C.), Saint Louis University, St Louis, Mo.

This work was supported by grants P50 33812 and HD25858 from the National Institute of Child Health and Human Development.

Paper previously presented at: Annual Meeting of the Organization for Human Brain Mapping, June 10-14, 2007; Chicago, III.

Please address correspondence to Todd Richards, Department of Radiology, Box 357115, Room AA010, Health Sciences Building, 1959 NE Pacific, Seattle, WA 98195; e-mail: toddr@u.washington.edu

DOI 10.3174/ajnr.A1007 left visual field resulting from a posterior callosal lesion and demonstrated how DTI can reveal the anatomic bases of a disconnection syndrome by tracking the degeneration of neural pathways and relating it to impaired fMRI activations and behavior. Dougherty et $\mathrm{al}^{4}$ used DTI to demonstrate that properties of specific fiber bundles match those of the hypothetical pathways described in the neurologic literature on alexia.

In the present study, we extend past research demonstrating fMRI connectivity differences between adults with and without dyslexia ${ }^{8}$ to test the hypothesis that these adults also differ in DTI structural changes (FA). DTI is an elegant method for measuring white matter structural changes to test for abnormalities in specific language pathways.

\section{Materials and Methods}

DTI scans were acquired from 7 healthy adult normal readers and from 14 adults with dyslexia on an Achieva 1.5T scanner (Philips, Best, The Netherlands). Participants were fathers in a family genetics study of dyslexia ${ }^{9,10}$; only fathers were studied, because they are less likely to compensate than mothers in this family genetics study. ${ }^{11}$ Informed consent was taken from all of the participants, and the study was approved by the University of Washington human subject committee (application 96-1872-D13).

The 7 right-handed control good readers and 14 right-handed dyslexic subjects were healthy, native English speakers and ranged in age from 30 to 45 years. The sample was drawn from a population that varied in education level, but most had some education beyond high school even if they did not complete a degree. All of the subjects completed a 4-hour phenotyping battery of psychometric measures, including the prorated verbal intelligence quotient (IQ) of the Wechsler Adult Intelligence Scale-Revised (WAIS-R). ${ }^{12}$ Measures were selected based on their good reliability and previous research showing that they assessed processes relevant to learning a written language. ${ }^{13,14}$ All of the fathers were parents of at least 1 child who met the research criteria for dyslexia. To be categorized as a good reader, the father did not show any indicator of dyslexia subphenotypes on a 
Table 1: Means, SDs, and values of $t$ comparing means on psychological measures for control subjects and adults with dyslexia

\begin{tabular}{|c|c|c|c|c|c|}
\hline \multirow[b]{2}{*}{ Measure } & \multicolumn{2}{|c|}{ Control } & \multicolumn{3}{|c|}{ Dyslexia } \\
\hline & Mean & SD & Mean & SD & $t$ \\
\hline Word Identification $\ddagger$ & 106.14 & 6.47 & 94.43 & 13.29 & $2.19^{*}$ \\
\hline Word Attack $\ddagger$ & 105.29 & 5.02 & 91.14 & 12.43 & $2.87 \dagger$ \\
\hline Real Word Reading & 101.71 & 11.60 & 85.07 & 9.95 & $3.42 \dagger$ \\
\hline Pseudo Word Reading & 94.43 & 18.17 & 77.07 & 9.49 & $2.91 \dagger$ \\
\hline GORT 3 Reading Accuracy§ & 14.86 & 2.61 & 9.00 & 4.61 & $3.10 \dagger$ \\
\hline GORT 3 Reading Rate§ & 14.00 & 2.24 & 9.00 & 3.26 & $3.63+$ \\
\hline WRAT 3 Spelling $\ddagger$ & 103.29 & 9.20 & 86.14 & 13.97 & $2.92 \dagger$ \\
\hline WIAT II Spelling $\ddagger$ & 113.00 & 12.38 & 94.85 & 13.04 & $3.02 \dagger$ \\
\hline Non Word Memory\|l & 1.09 & 0.88 & 0.70 & 0.67 & 1.13 \\
\hline RAN Letter\| & -0.14 & 0.80 & 1.60 & 1.43 & $-2.97 \dagger$ \\
\hline RAS Letter/Number\| & 0.10 & 1.00 & 2.41 & 1.51 & $-3.66 \dagger$ \\
\hline
\end{tabular}

Note:-WRMT-R word attack indicates the rate of pronouncing pseudowords on a list, Woodcock Reading Mastery Test-Revised; TOWRE sight word efficiency, rate of pronouncing single words on a list, Test of Word Reading Efficiency; TOWRE phonemic decoding efficiency, rate of pronouncing pseudowords on a list, Test of Word Reading Efficiency; GORT 3 accuracy, accuracy of pronouncing words during oral reading of text, Gray Oral Reading Test; GORT 3 rate, rate of pronouncing words during oral reading of text, Gray Oral Reading Test; WRAT 3 spelling and WIAT II spelling, written spelling of dictated words; nonword memory, oral reproduction of aurally presented pseudowords; RAN letter, rate of pronouncing randomly presented letters in rows; RAN letter and number, rate of pronouncing switching letters and numbers presented in rows.

$* P<.05$

$\dagger P<.01$

$\ddagger$ Scores on a scale of mean $=100 ; \mathrm{SD}=15$

$\S$ Scores on a scale of mean $=10 ; \mathrm{SD}=3$.

Z-scores on a scale of mean $=0 ; S D=1$

4-hour test battery ${ }^{13,14}$ and did not report any history of ever having reading or writing problems. To be categorized as a father with dyslexia, the father showed indicators of persisting dyslexia: their accuracy and/or rate in oral single pseudoword reading, single real word reading, or passage reading and/or spelling were below the population mean, and at least 1 SD below WAIS-R prorated verbal IQ; they also reported a history of reading and/or spelling problems. Table 1 reports the means and SDs for each measure for the dyslexic subjects and good readers. Woodcock Reading Mastery Test-Revised (WRMT-R $)^{15}$ Word Identification and Test of Word Reading Efficiency (TOWRE) ${ }^{16}$ Real Word Reading Efficiency assess the accuracy and rate, respectively, of real word reading. WRMT-R Word Attack and TOWRE Phonemic Decoding Efficiency assess the accuracy and rate, respectively, of phonologic decoding. Gray Oral Reading Test $3^{17}$ assesses accuracy and rate of oral reading of text.

The fathers did not differ significantly in mean WAIS-R prorated verbal IQ, a measure of ability to reason verbally. The affected fathers were significantly lower than the control fathers on each of the reading, spelling, and rapid automatic naming measures given, except phonologic working memory, for which the difference failed to reach conventional levels of statistical significance (Table 1). These results show that, despite comparable ability to reason verbally, the fathers with dyslexia had failed to develop comparable reading and writing skills and had persistent difficulty in rapidly associating phonologic codes with alphanumeric stimuli.

\section{MR Imaging Acquisition and Analysis}

DTI was performed by using a single-shot spin-echo diffusionweighted echo-planar pulse sequence with 64 sections covering the whole brain at 2.5-mm section thickness ( TR/TE $=9500 / 74 \mathrm{~ms}$; acquisition matrix $=128 \times 128)$. Diffusion MR images were obtained from 32 noncolinear directions with a b-value of $1000 \mathrm{~s} / \mathrm{mm}^{2}$ along with a $b=0$ image with no diffusion gradients. Images were processed off-line by using FSL (FMRIB [The Oxford Centre for Functional Magnetic Resonance Imaging of the Brain] Software Library, http:// www.fmrib.ox.ac.uk/fsl), which included eddy-current compensation, DTIFit to reconstruct diffusion tensors, and FA. DTIFit is FSL software that fits a diffusion tensor model at each voxel of the diffusion images. DTIFit generates the following $3 \mathrm{D}$ images at the same matrix size and resolution as the original diffusion images: V1 (first eigenvector), V2 (second eigenvector), V3 (third eigenvector), L1 (first eigenvalue), L2 (second eigenvalue), L3 (third eigenvalue), mean diffusivity, FA, and S0 (raw T2 signal with no diffusion weighting). Voxelwise statistical analysis of the FA data were carried out by using tract-based spatial statistics (TBSS), ${ }^{18}$ part of FSL. ${ }^{19}$ First, FA images were created by fitting the diffusion tensor to the raw diffusion data by using (FMRIB Diffusion Toolbox) and then were brain extracted by using brain extraction tool (BET), part of FSL. ${ }^{20}$ All of the subjects' FA data were then aligned into a common space by using the nonlinear registration IRTK ${ }^{21}$ (Image Registration Toolkit; http:// www.doc.ic.ac.uk/ dr/software). The mean FA image was then created and thinned to create a mean FA skeleton, which represents the centers of all tracts common to the group. The TBSS method does not need/perform any presmoothing.

In more detail, the coregistration/skeletonization process involves the following steps. ${ }^{18}$ First, all of the subjects' FA images are affinetransformed into $1 \times 1 \times 1 \mathrm{~mm}^{3} \mathrm{MNI} 152$ space (a normalized/averaged brain developed by the Montreal Neurological Institute). Second, the transformed FA images are averaged to create a mean FA image. Third, the mean FA is fed into the tract skeleton generation, which aims to represent all of the tracts that are "common" to all of the subjects. The skeleton will represent each such tract as a single line (or surface) running down the center of the tract. To achieve skeletonization, the local surface perpendicular direction is estimated (at all of the voxels in the image), and a nonmaximum suppression in this direction is performed. In other words, a search is made along all of the voxels in the local "tract perpendicular direction," and the voxel with the highest FA is identified as the center of the tract. The estimated tract perpendicular direction is regularized to improve estimation robustness. Fourth, the center of each tract is found by comparing the FA value with the 2 closest neighbors on each side, in the direction of the tract perpendicular. If the FA value is greater than the neighboring values, then the voxel is marked as lying on the skeleton.

Each subject's aligned FA data were then projected onto this skeleton (Fig 1), and the resulting data were fed into voxelwise crosssubject statistics. A randomization procedure (FSL's randomize, Monte Carlo permutation test) was used to perform the group analysis statistics. TBSS group maps were generated for the nonparamet- 


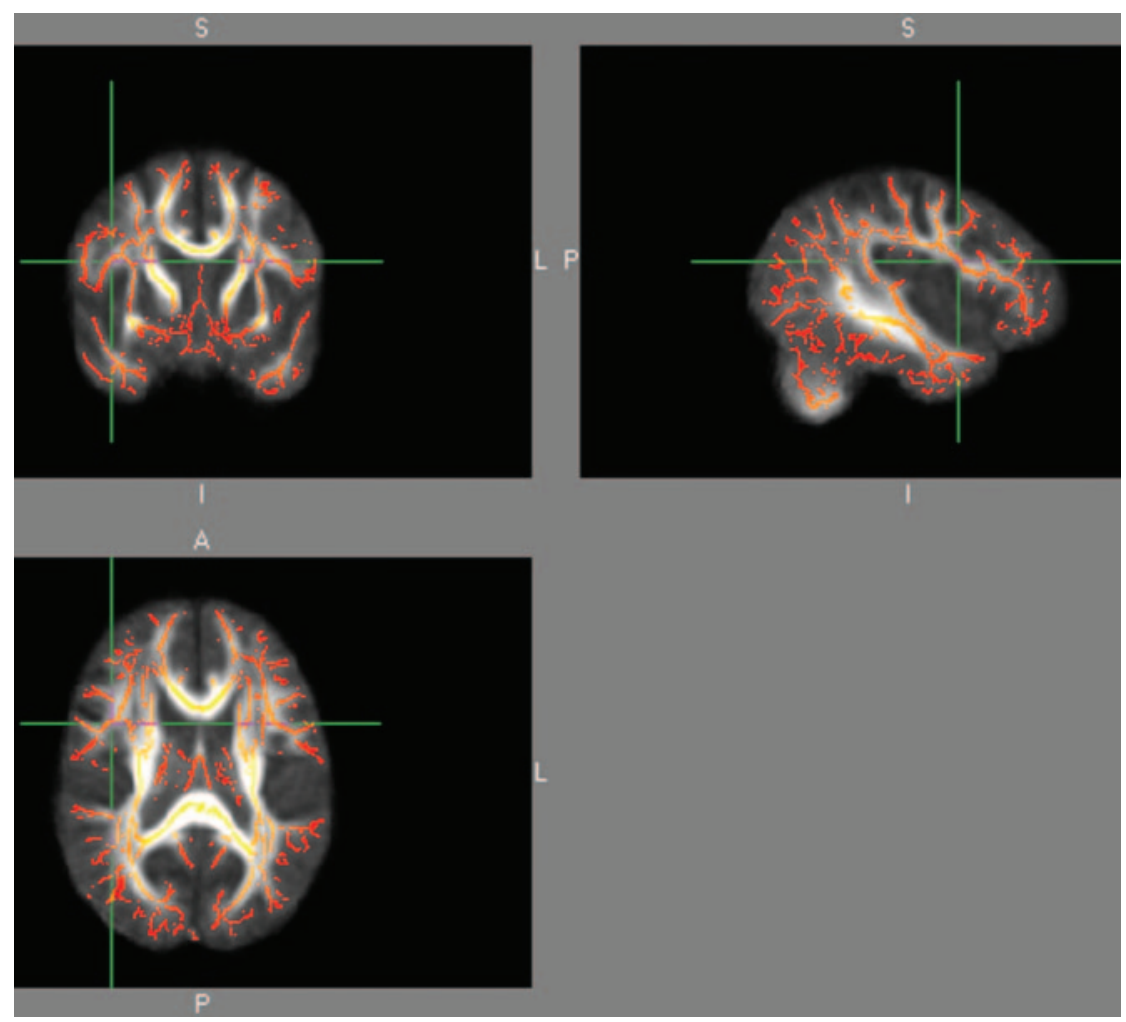

Fig 1. Overlay of skeletonized averaged FA map (orange) onto standardized FA map from DTIs.

ric 2-sample unpaired $t$ test to test whether the control group was greater than dyslexic group and to test whether the dyslexic group was greater than the control group. To perform this nonparametric 2-sample $t$ test, 4D images were created of all of the coregistered skeletonized FA maps (from all of the subjects) and then the following command was executed: "randomize -i TwoSamp4D -o TwoSampT -d design.mat -t design.con -m mask - c 3." Where TwoSamp4D is the input $4 \mathrm{D}$ data of all of the subjects, design.mat contains the design matrix for the GLM model, design.con contains the between group contrast matrix to use with the design matrix, and mask is a brain mask based on FSL's standard brain mask (avg152_brain_mask.hdr/. img). Randomize produces a test statistic image (eg, tstat1) and sets of $P$ value images (stored as $1-\mathrm{P}$ for more convenient visualization). The $-\mathrm{c} 3$ options allowed for cluster-based thresholding ( $t$ value threshold of 3 in this case) corrected for multiple comparisons by using the null distribution of the maximum (across the image) cluster size, and the $-\mathrm{c}$ options outputs a $3 \mathrm{D}$ image showing the exact location of only the significant clusters with zeros everywhere else. All of the statistical output images are in NIFTI GZ (Neuroimaging Informatics Technology Initiative gzipped) format and were viewed and overlaid onto the standard brain using FSLVIEW (FSL software tool). To anatomically define the significant clusters, the DTI maps were coregistered both to the AAL atlas (automated anatomical labeling map which is a $3 \mathrm{D}$ map containing 116 brain regions co-registered to standard MNI space developed by Tzourio-Mazoyer et $\mathrm{al}^{22}$; http:// www.sph.sc.edu/comd/rorden/template.html) and to a DTI atlas (http://www.loni.ucla.edu/Atlases/Atlas_Detail.jsp?atlas_id = 15). Custom software was written in Fortran to identify the brain region correspondence and MNI (Montreal Neurological Institute) coordinates of each significant cluster.

The authors acknowledge that the TBSS technique has limitations with regard to measuring FA values in regions where there are crossing tracts or tract junctions. Voxelwise statistics are still difficult to estimate and interpret at tract junctions or crossings. Skeleton contiguity is not enforced at junctions, and an artificial lowering of FA value can occur at these junctions or crossing tracts. Another potential confound could have been caused by head motion; however, both dyslexic and control subjects were not different in motion (control $X$ direction movement in pixels: mean $\pm \mathrm{SD}=0.22 \pm 0.05$; dyslexic $\times$ direction movement: mean $\pm \mathrm{SD}=0.23 \pm 0.045 ; t=0.56 ; P=.59$; control y direction movement in pixels: mean $\pm \mathrm{SD}=0.31 \pm 0.11$; dyslexic y direction movement: mean $\pm \mathrm{SD}=0.30 \pm 0.067 ; t=0.15$; $P=.88$ ) as measured by a centroid calculation of the 33 DTI volumes acquired sequentially.

\section{Results}

The skeletonized TBSS group map statistical tests showed that control subjects had greater FA compared with dyslexic subjects in many language-related white matter fiber tracts in these adults (Fig 2 and Tables 2 and 3). Significant group difference map clusters occurred in 28 regions located in bilateral white matter tracts within the frontal lobe, temporal lobe, occipital lobe, and parietal lobe (Table 2, as measured from the AAL atlas boundaries). Significant group difference map clusters occurred in an additional 7 regions located in white matter tracts not defined by the AAL atlas (Table 3, as measured from the DTI atlas boundaries).

For test of dyslexic subjects greater than control subjects for the FA value, there were 9 regions with significant clusters (Table 4, as measured from the AAL atlas boundaries). An additional significant group difference map cluster occurred in 1 cerebellar region located in white matter tracts not defined by the AAL atlas (Table 5, as measured from the DTI atlas boundaries). Although some of these regions appear to overlap with the control regions, there were no FA voxels that overlap with the test of control subjects greater than dyslexic 


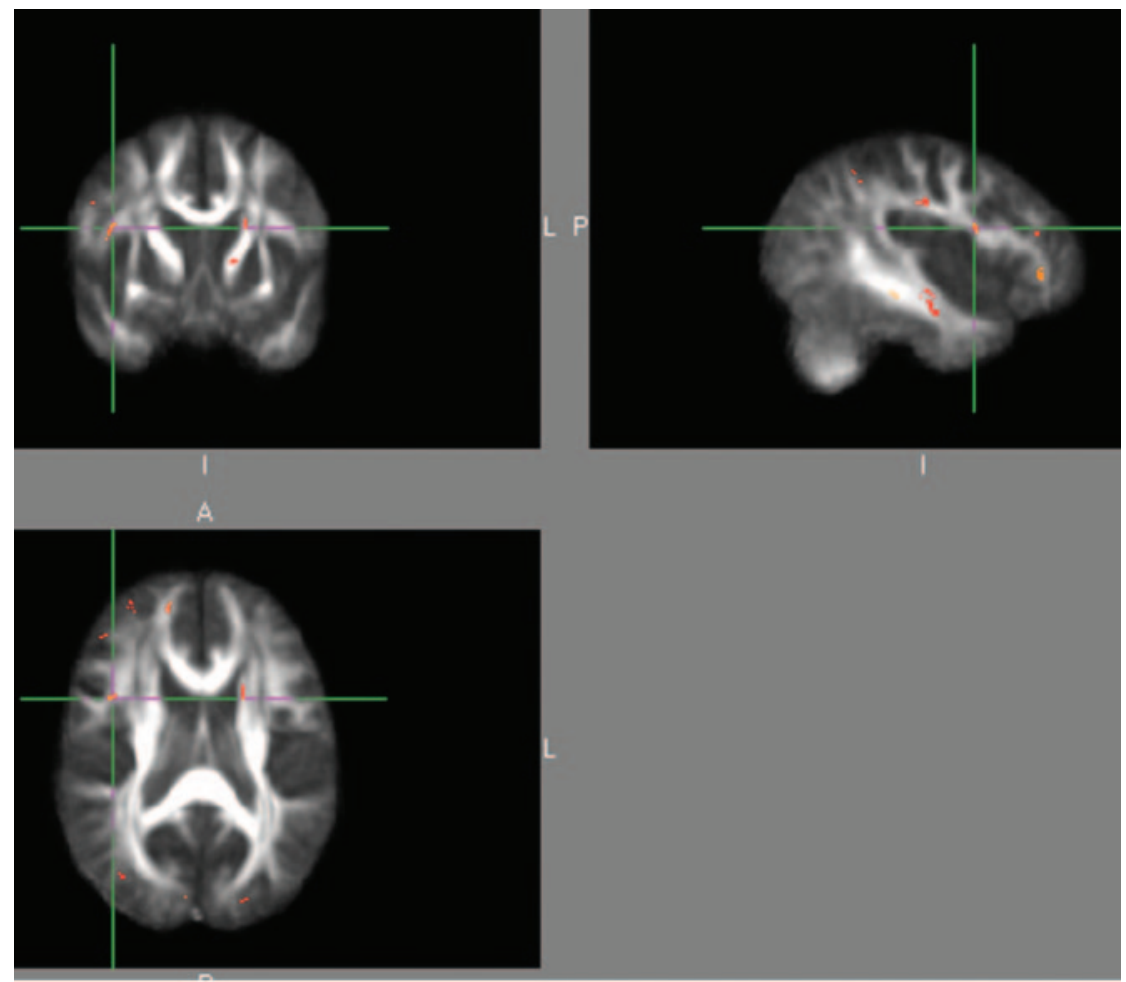

Fig 2. Group difference map for control subjects greater than dyslexic subjects for skeletonized diffusion FA using TBSS software. The crosshair is positioned on a significant cluster near the right inferior frontal gyrus. Orange-red areas show significant clusters for group difference.

subjects. In other words, the clusters for this test are spatially separated from the control greater than dyslexic clusters. The largest clusters were in the cerebellum for this test, and the control subjects greater than dyslexic subjects had no clusters in the cerebellum.

Software was written to extract individual subject DTI FA

\begin{tabular}{|c|c|c|c|c|c|c|c|}
\hline \multirow[b]{2}{*}{ Brain Region } & \multirow{2}{*}{$\begin{array}{c}t \\
\text { Statistic }\end{array}$} & \multirow{2}{*}{$\begin{array}{l}\text { No. of } \\
\text { Voxels }\end{array}$} & \multicolumn{3}{|c|}{ MNI Coordinatest } & \multirow{2}{*}{$\begin{array}{c}\text { Brodmann } \\
\text { Area }\end{array}$} & \multirow{2}{*}{$\begin{array}{l}\text { Stanberry } \\
\text { Comparison }\end{array}$} \\
\hline & & & $\mathrm{x}$ & y & z & & \\
\hline Precentral L & 2.55 & 42 & -35 & -7 & 38 & 6 & No \\
\hline Precentral R & 2.94 & 88 & 52 & 0 & 21 & 6 & No \\
\hline Frontal Sup R & 2.97 & 54 & 19 & 55 & 17 & 10 & Yes \\
\hline Frontal Mid R & 2.81 & 45 & 36 & 36 & 17 & 46 & Yes \\
\hline Frontal Inf Oper R & 2.80 & 86 & 55 & 19 & 0 & 44 & Yes \\
\hline Frontal Inf Tri R & 2.80 & 38 & 38 & 43 & 1 & 45 & Yes \\
\hline Frontal Inf Orb L & 3.02 & 60 & -47 & 38 & -12 & 47 & No \\
\hline Frontal Inf Orb R & 2.74 & 91 & 51 & 36 & -8 & 47 & No \\
\hline Supp Motor Area R & 2.95 & 44 & 14 & 3 & 61 & 6 & No \\
\hline Cuneus $R$ & 2.89 & 59 & 9 & -94 & 12 & 18 & No \\
\hline Lingual L & 2.84 & 49 & -10 & -89 & -17 & 18 & No \\
\hline Occipital Sup L & 2.77 & 31 & -20 & -90 & 18 & 18 & No \\
\hline Occipital Sup R & 2.78 & 36 & 26 & -76 & 26 & 7 & No \\
\hline Occipital Mid R & 2.92 & 51 & 42 & -77 & 18 & 7 & Yes \\
\hline Fusiform L & 2.90 & 21 & -20 & -84 & -19 & 18 & Yes \\
\hline Fusiform R & 2.76 & 81 & 41 & -11 & -32 & 20 & Yes \\
\hline Postcentral L & 3.12 & 26 & -52 & -19 & 30 & 48 & No \\
\hline Postcentral R & 2.53 & 27 & 53 & -1 & 22 & 3 & No \\
\hline Parietal Sup L & 3.10 & 42 & -14 & -72 & 47 & 5 & No \\
\hline Parietal Inf $\mathrm{L}$ & 2.68 & 28 & -30 & -43 & 45 & 40 & No \\
\hline Parietal Inf $\mathrm{R}$ & 3.31 & 41 & 47 & -47 & 40 & 40 & Yes \\
\hline Precuneus L & 2.76 & 16 & -10 & -72 & 48 & 7 & No \\
\hline Putamen L & 2.84 & 32 & -17 & 11 & 2 & 0 & No \\
\hline Temporal Sup L & 2.69 & 48 & -48 & -33 & 8 & 22 & No \\
\hline Temporal Pole Sup L & 2.88 & 32 & -23 & 15 & -31 & 38 & No \\
\hline Temporal Mid L & 3.08 & 69 & -47 & -34 & 8 & 39 & No \\
\hline Temporal Inf L & 2.79 & 104 & -60 & -54 & -19 & 37 & Yes \\
\hline Temporal Inf R & 2.57 & 27 & 41 & -9 & -33 & 20 & Yes \\
\hline
\end{tabular}

Note:-The last column shows the regions where both the current study and Stanberry et al ${ }^{8}$ functional connectivity study have consistency; Sup, superior; Inf, inferior; Orb, orbital; Supp, supplementary; Oper, operculum; L, left; R, right.

* AAL refers to the Automated Anatomical Labeling map which is a 3-dimensional map containing 116 brain regions co-registered to standard MNI space.

$\dagger \mathrm{MNI}$ coordinates refers to a standard brain imaging coordinate system developed by the Montreal Neurological Institute. 
Table 3: White matter brain regions within the boundaries of the DTI tract atlas regions where control subjects were significantly greater than dyslexic subjects in the skeletonized fractional anisotropy

\begin{tabular}{|c|c|c|c|c|c|}
\hline \multirow[b]{2}{*}{ White Matter Tract Name } & \multirow{2}{*}{$\begin{array}{c}t \\
\text { Statistic }\end{array}$} & \multirow{2}{*}{$\begin{array}{l}\text { No. of } \\
\text { Voxels }\end{array}$} & \multicolumn{3}{|c|}{ MNI Coordinates } \\
\hline & & & $\mathrm{x}$ & $\mathrm{y}$ & z \\
\hline Body of corpus callosum & 2.63035 & 13 & -14 & -1 & 36 \\
\hline Anterior limb of internal capsule left & 2.88289 & 48 & -17 & 11 & 2 \\
\hline Superior corona radiata left & 3.11258 & 11 & -16 & -4 & 40 \\
\hline Posterior corona radiata left & 2.74872 & 21 & -18 & -47 & 33 \\
\hline Sagittal stratum & 2.62251 & 32 & -46 & -28 & -12 \\
\hline Superior longitudinal fasciculus left & 2.70963 & 55 & -37 & -23 & 32 \\
\hline Superior fronto-occipital fasciculus & 3.04868 & 26 & -21 & 9 & 20 \\
\hline
\end{tabular}

Note:-DTI indicates diffusion tensor imaging; MNI coordinates, standard brain imaging coordinate system developed by the Montreal Neurologial Institute.

Table 4: White matter regions within the boundaries of the AAL* atlas where dyslexic subjects were significantly greater than control subjects in the skeletonized fractional anisotropy

\begin{tabular}{|c|c|c|c|c|c|c|}
\hline \multirow[b]{2}{*}{ Brain Region } & \multirow{2}{*}{$\begin{array}{c}t \\
\text { Statistic }\end{array}$} & \multirow{2}{*}{$\begin{array}{l}\text { No. of } \\
\text { Voxels }\end{array}$} & \multicolumn{3}{|c|}{ MNI Coordinatest } & \multirow{2}{*}{$\begin{array}{c}\text { Brodmann } \\
\text { Areas } \\
\end{array}$} \\
\hline & & & $\mathrm{x}$ & $\mathrm{y}$ & $\mathrm{z}$ & \\
\hline Precentral L & 2.61863 & 23 & -44 & 8 & 33 & 44 \\
\hline Frontal Inf Orb L & 2.81102 & 21 & -39 & 26 & -14 & 47 \\
\hline Fusiform R & 2.90205 & 19 & 37 & -41 & -24 & 37 \\
\hline Postcentral L & 2.89957 & 15 & -47 & -23 & 45 & 3 \\
\hline Temporal Mid L & 2.80589 & 36 & -54 & 2 & -32 & 20 \\
\hline Temporal Mid R & 2.84047 & 16 & 61 & -11 & -16 & 21 \\
\hline Temporal Inf L & 2.89029 & 39 & -45 & -21 & -33 & 20 \\
\hline Cerebellum R & 2.68122 & 116 & 41 & -60 & -39 & \\
\hline Cerebellum L & 2.76867 & 45 & -42 & -63 & -48 & \\
\hline
\end{tabular}

Note:- L, indicates left; R, right; Inf, inferior; Orb, orbital.

* AAL refers to the automated anatomical labeling map which is a 3D map containing 116 brain regions co-registered to standard MNI space.

† MNI coordinates refers to a standard brain imaging coordinate system developed by the Montreal Neurological Institute.

Table 5: White matter brain regions within the boundaries of the DTI tract atlas regions where dyslexic subjects were significantly greater than control subjects in the skeletonized fractional anisotropy

\begin{tabular}{|c|c|c|c|c|c|}
\hline \multirow[b]{2}{*}{ White matter tract name } & \multirow{2}{*}{$\begin{array}{c}t \\
\text { Statistic }\end{array}$} & \multirow{2}{*}{$\begin{array}{l}\text { No. of } \\
\text { Voxels }\end{array}$} & \multicolumn{3}{|c|}{ MNI Coordinates } \\
\hline & & & $\mathrm{x}$ & $\mathrm{y}$ & z \\
\hline Middle cerebellar peduncle & 2.796 & 31 & -33 & -52 & -42 \\
\hline
\end{tabular}

Note:-DTI indicates diffusion tensor imaging; MNI, MNI coordinates, standard brain imaging coordinate system developed by the Montreal Neurological Institute.

and eigen values within a significant cluster near the left inferior frontal gyrus. The results are shown in Table 4 . There was a significant difference $(t$ test $P<.005)$ between the control and dyslexic subjects as tested in these individual FA values in column 2.

\section{Discussion}

These results demonstrate white matter differences between adults with and without dyslexia in language-related regions of the brain (see specific region description below). Similar to the results reported by Stanberry et $\mathrm{al}^{8}$ that showed stronger functional connectivity in adult control subjects from seed points placed in both bilateral inferior frontal gyri, the current study group also showed differences in the inferior frontal gyri along with several additional brain regions. These results demonstrate DTI structural connectivity that is consistent with the functional connectivity results (Table 2). Common brain regions found across structural and functional connectivity studies include the right superior frontal gyrus, right middle frontal gyrus, right inferior frontal gyrus-operculum, right inferior frontal gyrus-pars triangularis, right middle occipital gyrus, bilateral fusiform gyri, right inferior parietal, and bilateral inferior temporal gyrus. In contrast to the previous DTI studies of developmental dyslexia that mainly showed differences in left temporoparietal brain regions, the current study reports DTI white matter differences (FA) between adults with

\begin{tabular}{|c|c|c|c|c|}
\hline \multirow{2}{*}{$\begin{array}{l}\text { Subject } \\
\text { Identification }\end{array}$} & \multirow[b]{2}{*}{ FA } & \multicolumn{3}{|c|}{ Eigenvalues $\times 1 \mathrm{e} 6$} \\
\hline & & $\lambda \mathrm{L} 1$ & $\lambda \mathrm{L} 2$ & $\lambda \mathrm{L} 3$ \\
\hline con 101 & 4096 & 1069 & 642 & 403 \\
\hline con 105 & 4180 & 1016 & 733 & 330 \\
\hline con 109 & 4059 & 1032 & 790 & 337 \\
\hline con 2658 & 4345 & 1190 & 665 & 440 \\
\hline con 3126 & 3878 & 1040 & 810 & 369 \\
\hline con 3131 & 5003 & 1158 & 699 & 359 \\
\hline con 3139 & 4262 & 1027 & 792 & 379 \\
\hline dys 103 & 3380 & 1161 & 768 & 549 \\
\hline dys 106 & 3659 & 986 & 744 & 387 \\
\hline dys 2253 & 3027 & 1004 & 723 & 503 \\
\hline dys 2254 & 3225 & 1102 & 845 & 502 \\
\hline dys 2291 & 3944 & 957 & 731 & 333 \\
\hline dys 2337 & 3220 & 1010 & 828 & 454 \\
\hline dys 2403 & 3877 & 1095 & 767 & 411 \\
\hline dys 2418 & 3033 & 1034 & 813 & 496 \\
\hline dys 2419 & 3668 & 1085 & 824 & 423 \\
\hline dys 2421 & 3813 & 1038 & 780 & 389 \\
\hline dys 2506 & 3746 & 987 & 721 & 387 \\
\hline dys 3021 & 4686 & 1122 & 652 & 405 \\
\hline dys 3053 & 3290 & 1031 & 790 & 457 \\
\hline dys 3198 & 4115 & 1046 & 752 & 410 \\
\hline
\end{tabular}

Note:-DTI indicates diffusion tensor imaging; FA, fractional anisotropy; con, control; dys, dyslexic. 
and without dyslexia not only in both temporal regions (fusiform and inferior temporal) but also in right parietal (inferior), right occipital (middle), and many frontal regions.

One possible reason for these reported differences with previous DTI studies is that the current study is the first study that used the new technique of the FSL tractography-based (skeletonized) method for coregistration and group map contrast statistical comparison in the study of dyslexia. However, the main result reported here is consistent with other dyslexia DTI studies (discussed in the introduction) showing lower FA values in white matter in dyslexic subjects compared with control subjects. A possible explanation for the lower FA values in dyslexia is that, in white matter, there could be a less coherent bundle of tracts relating to connectivity of these important language pathways. In other words, the directionality and number of fibers in the white matter may be different in dyslexia and relate to how the water diffuses more readily along the orientation of axonal fibers than in other directions due to obstruction from structural components, such as the myelin sheath. Of particular note is the important change found in the superior longitudinal fasciculus (SLF), which has a role in language. The SLF is composed of 4 distinct components, ${ }^{23}$ SLF I, SLF II, SLF III, and arcuate fascicle. SLF III connects the rostral inferior parietal cortex, which receives information from the ventral precentral gyrus. This suggests that the SLF III transfers somasensory information, such as language articulation, between the ventral premotor cortex, the Brodmann 44 (pars opercularis), the supramarginal gyrus (Brodmann 40), and the lateral inferior prefrontal cortex working memory (Brodmann 46).

\section{Conclusion}

The DTI FA results in the bilateral white matter showing higher FA in adult control subjects compared with adults with dyslexia (relating to white matter fiber tract integrity) are consistent with our previous functional connectivity results from seed points in the bilateral inferior frontal gyrus. These DTI findings add to previous MR imaging, functional magnetic resonance spectroscopy, and fMRI connectivity differences found in the right inferior frontal gyrus regions between people with and without dyslexia, ${ }^{24-26}$ which suggest that anomalies at many levels of the brain substrate in this region may contribute to dyslexia. Taken together, these results suggest disconnections in structural, as well as functional, connectivity in the development of dyslexia.

\section{References}

1. Stegemann T, Heimann M, Dusterhus P, et al. Diffusion tensor imaging (DTI) and its importance for exploration of normal or pathological brain development. Fortschr Neurol Psychiatr 2006;74:136-48
2. Le Bihan D, Mangin JF, Poupon C, et al. Diffusion tensor imaging: concepts and applications. J Magn Reson Imaging 2001;13:534-46

3. Deutsch GK, Dougherty RF, Bammer R, et al. Children's reading performance is correlated with white matter structure measured by diffusion tensor imaging. Cortex 2005;41:354-63

4. Dougherty RF, Ben-Shachar M, Deutsch G, et al. Occipital-callosal pathways in children: validation and atlas development. Ann NY Acad Sci 2005; $1064: 98-112$

5. Klingberg T, Hedehus M, Temple E, et al. Microstructure of temporo-parietal white matter as a basis for reading ability: evidence from diffusion tensor magnetic resonance imaging. Neuron 2000;25:493-500

6. Molko N, Cohen L, Mangin JF, et al. Visualizing the neural bases of a disconnection syndrome with diffusion tensor imaging. J Cogn Neurosci 2002;14:629-36

7. Niogi SN, McCandliss BD. Left lateralized white matter microstructure accounts for individual differences in reading ability and disability. Neuropsychologia 2006;44:2178-88

8. Stanberry LI, Richards T, Berninger VW, et al. Low frequency signal changes reflect differences in functional connectivity between good readers and dyslexics during continuous phoneme mapping. Magn Reson Imaging 2006;24:217-29

9. Raskind W, Hsu L, Berninger V, et al. Familial aggregation of dyslexic phenotypes. Behav Genet 2000;30:385-96

10. Raskind W, Igo R, Chapman N, et al. A genome scan in multigenerational families with dyslexia: Identification of a novel locus on chromosome $2 \mathrm{q}$ that contributes to phonological decoding efficiency. Mol Psychiatry 2005;10:699-711

11. Raskind WH. Current understanding of the genetic basis of reading and spelling disability. J Learn Disabil Q 2001;24:141-57

12. Wechsler D. Wechsler Adult Intelligence Scale-Revised (WAIS-R). San Antonio, Tex: Psychological Corporation; 1981

13. Berninger V, Abbott R, Thomson J, et al. Modeling developmental phonological core deficits within a working memory architecture in children and adults with developmental dyslexia. Sci Studies Reading 2006;10:165-98

14. Berninger V, Abbott R, Thomson J, et al. Language phenotype for reading and writing disability: A family approach. Sci Studies Reading 2001;5:59-105

15. Woodcock R. Woodcock Reading Mastery Test - Revised. Circle Pine, Minn: American Guidance Service; 1987

16. Torgesen J, Wagner R, Rashotte C. Test of Word Reading Efficiency. Austin, Tex: ProEd; 1999

17. Wiederholt J, Bryant B. Gray Oral Reading Test. 3rd ed. Odessa, Fla: Psychological Assessment Resources; 1992

18. Smith SM, Jenkinson M, Johansen-Berg H, et al. Tract-based spatial statistics: voxelwise analysis of multi-subject diffusion data. Neuroimage 2006;31: $1487-505$

19. Smith SM, Jenkinson M, Woolrich MW, et al. Advances in functional and structural MR image analysis and implementation as FSL. Neuroimage 2004; 23(suppl 1):S208-S219

20. Smith SM. Fast robust automated brain extraction. Hum Brain Mapp 2002;17:143-55

21. Rueckert D, Sonoda LI, Hayes C, et al. Non-rigid registration using free-form deformations: application to breast MR images. IEEE Trans Med Imaging 1999;18:712-21

22. Tzourio-Mazoyer N, Landeau B, Papathanassiou D, et al. Automated anatomical labeling of activations in SPM using a macroscopic anatomical parcellation of the MNI MRI single-subject brain. Neuroimage 2002;15:273-89

23. Makris N, Kennedy DN, McInerney S, et al. Segmentation of subcomponents within the superior longitudinal fascicle in humans: a quantitative, in vivo, DT-MRI study. Cereb Cortex 2005; 15:854-69

24. Richards T, Aylward E, Berninger V, et al. Individual fMRI activation in orthographic mapping and morpheme mapping after orthographic or morphological spelling treatment in child dyslexics. J Neurolinguistics 2006;19:56-86

25. Richards T, Aylward E, Raskind W, et al. Converging evidence for triple word form theory in child dyslexia. Dev Neuropsychol 2006;30:547-90

26. Berninger $\mathrm{V}$, Raskind $\mathrm{W}$, Richards $\mathrm{T}$, et al. A multidisciplinary approach to understanding developmental dyslexia within working-memory architecture: genotypes, phenotypes, brain, and instruction. Dev Neuropsychol 2007; In Press 